\title{
A CLASS OF SPACES AND THEIR ANTI SPACES
}

\author{
C. K. BASU
}

\begin{abstract}
The present paper is a continuation of the study of $S$-closed and $s$-closed topological spaces as introduced by Thompson [11] and Maio and Noiri [7] respectively. Although there is no relation between compactness with $S$-closedness or $s$-closedness, this paper yields some new characterizations of these concepts in terms of compactness.
\end{abstract}

\section{Introudction}

Since the introduction of semi-open sets by N. Levine [6], many mathematicians have introduced many new topological properties, using semi-open sets. Maio and Noiri [7] initiated the study of a class of topological spaces under the terminology " $s$-closed spaces", which is properly contained in the class of $S$-closed spaces as introduced by Thompson [11] and subsequently studied extensively by many mathematicians. Ganster and Reilly [4] have shown a remarkable result towards the distinction between these concepts that every infinite topological space can be represented as a closed subspace of a connected $S$-closed space which is not $s$-closed. The aim of this paper is to study these topological properties viz. $S$-closedness and $s$-closedness via compactness which reflect the distinction between the concepts of compactness and $S$-closeness or $s$-closedness. This, however, leads us to establish in a straight forward manner certain important characterization theorems of $S$-closed spaces and $s$-closed spaces which are already wellknown. In the last section, we introduce and characterize the class of anti- $S$-closed and anti- $s$-closed spaces.

By $(X, T)$ or simply by $X$ we shall denote a topological space, and for a subset $A$ of $X$, the closure of $A$ and the interior of $A$ will be denoted by $\mathrm{cl} A$ and int $A$ respectively. A subset $A$ of $X$ is said to be semi-open [6] if there exists an open set $U$ of $X$ such that $U \subset A \subset \operatorname{cl} U$. Biswas [2] used semi-open sets to define semi-closed sets and semi-closure of a set. A subset $A$ of $X$ is semi-closed iff $X-A$ is semi-open and the semi-closure of $A$, denoted by $\operatorname{scl} A$, is the intersection of all semi-closed sets containing $A$ [2]. A set which is semi-open as well as semi-closed is said to be a semi-regular set [7]. Maio and Noiri [7] characterized semi regular sets in terms of regular open sets as follows: a set

Received May 29, 2002; revised September 26, 2002.

2000 Mathematics Subject Classification. 54D99.

Key words and phrases. $S$-sets, $s$-sets, semi-regular sets, regular closed sets, $s$-accumulation point, SR-accumulation point, $(\theta, s)$ continuous function, $\gamma$-continuous function, anti- $S$-closed and anti-s-closed spaces. 
$A$ is semi-regular iff there exists a regular open set $U$ of $X$ such that $U \subset A \subset \operatorname{cl} U$. The family of all semi-open (resp. semi-regular, regular-open, regular closed) sets of $X$ will be denoted by $\mathrm{SO}(X)$ (resp. $\mathrm{SR}(X), \mathrm{RO}(X), \mathrm{RC}(X)$ ). While the collection of all members of $\mathrm{SO}(X)$ (resp. $\mathrm{SR}(X), \mathrm{RO}(X), \mathrm{RC}(X)$ ) each containing a point $x$ of $X$ will be denoted by $\mathrm{SO}(x)$ (resp. $\mathrm{SR}(x), \mathrm{RO}(x), \mathrm{RC}(x)$ ). A subset $A$ of $X$ is said to be $S$-closed [9] (resp. $s$-closed [7]) relative to $X$ or simply an $S$-set (s-set) iff every cover of $A$ by sets of $\mathrm{SO}(X)$ admits a finite subfamily whose closures (resp. semi-closures) cover $A$. In case $A=X$ and $A$ is an $S$-set (s-set), then $X$ is called an $S$-closed [11] (resp. s-closed [7]) space.

\section{S-Closed and s-Closed Spaces}

Analogous to a well known theorem on compactness, Asha Mathur [8] and Maio and Noiri [7] respectively proved that a topological space $X$ is $S$-closed (resp. $s$-closed) iff every regular closed (resp. semi-regular) cover of $X$ has a finite subcover. Although it is well known that compactness and $S$-closedness (resp. $s$-closedness) are independent notions, it is our intention in this section to study such spaces with the help of compactness. An important and useful consequence of such study is to achieve a new approach which not only simplifies (in a straightforward way) the proofs of some wellknown characterization theorems of $S$-closed and $s$-closed spaces but also improves some characterization theorem of such spaces. Joseph and Kwack [5] and Ganguly and Basu [3] initiated respectively $(\theta, s)$-continuous function and $\gamma$-continuous function to study $S$-closed (resp. $s$-closed) spaces. Using those functions, we derive that a topological space $X$ is $S$-closed (resp. $s$-closed) iff it is a $(\theta, s)$-continuous (resp. $\gamma$-continuous) image of a compact space. For these purposes we require some definitions and results.

Definition 2.1. A filter base $\Im$ on $X$ is said to $s$-accumulate [11] (resp. SRaccumulate [7]) to $x \in X$ iff for each $V \in \mathrm{SO}(x)$ and each $F \in \Im$ satisfy $F \cap \operatorname{cl} V \neq \phi$ (resp. $F \cap \operatorname{scl} V \neq \phi)$.

Joseph and Kwack [5] and Maio and Noiri [7] respectively established that $\mathrm{RC}(x)=$ $\{\operatorname{cl} V: V \in \mathrm{SO}(x)\}$ and $\operatorname{SR}(x)=\{\operatorname{scl} V: V \in \mathrm{SO}(x)\}$. Therefore an equivalent formulation of the above definition is that a filter base $\Im$ on $X$ is said to have an $s$ accumulation (resp. SR-accumulation) point $x$ iff for each $F \in \Im$ and for each $V \in \operatorname{RC}(x)$ (resp. $V \in \mathrm{SR}(x)), F \cap V \neq \phi$.

Definition 2.2. A filter base $\Im$ on $X$ is said to $s$-converge [11] (resp. SR-converge [7]) to $x$ iff for each $V \in \mathrm{RC}(x)$ (resp. $V \in \mathrm{SR}(x)$ ) there is an $F \in \Im$ satisfying $F \subset V$.

The corresponding definitions for nets are obvious.

Definition 2.3. Let $(X, T)$ be a topological space. We define $T_{\mathrm{RC}}$-topology (resp. $T_{\mathrm{SR}}$-topology) on $X$ as the topology on $X$ which has $\mathrm{RC}(X)$ (resp. $\left.\mathrm{SR}(X)\right)$ as a subbase. It is to be noted that intersection of even two regular closed (resp. semi-regular) sets may 
fail to be regular closed (resp. semi-regular). Therefore these collections do not form a base for topology.

Definition 2.4. A filter base $\Im$ in $(X, T)$ is said to be $T_{\mathrm{RC}}$-convergent (resp. $\mathrm{T}_{\mathrm{SR}^{-}}$ convergent) to $x$ if $\Im$ converges to $x$ in $\left(X, T_{\mathrm{RC}}\right)$ (resp. in $\left(X, T_{\mathrm{SR}}\right)$ ).

Proposition 2.5. A filterbase $\Im$ in $(X, T)$ s-converges (resp. SR-converges) to $x$ iff

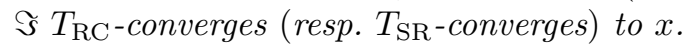

Proof. Straightforward.

The corresponding proposition using nets is also obvious.

Definition 2.6. A filter base $\Im$ on $(X, T)$ is said to have $x$ as a $T_{\mathrm{RC}}$-accumulation (resp. $T_{\mathrm{SR}}$-accumulation) point if $x$ is an accumulation point of $\Im$ in $\left(X, T_{\mathrm{RC}}\right)$ (resp. in $\left.\left(X, T_{\mathrm{SR}}\right)\right)$.

Similarly, $T_{\mathrm{RC}}\left(\right.$ resp. $T_{\mathrm{SR}}$ )-accumulation point of a net can be defined.

Remark 2.7. Every $T_{\mathrm{RC}}$-accumulation (resp. $T_{\mathrm{SR}}$-accumulation) point of a filter or a net is also an $s$-accumulation (resp. SR-accumulation) point. But the converse is not necessarily true follows from the following example.

Example 2.8. Let $X=R$, be the set of reals with the usual topology then $\left(X, T_{\mathrm{RC}}\right)$ (resp. $\left(X, T_{\mathrm{SR}}\right)$ ) is clearly the discrete topology. Let $x_{n}=(-1)^{n} .1 / n$ for each positive integer $n$, then the net $\left\{x_{n}\right\}_{n \in N}$ and the filter $\Im$ based on the net $\left\{x_{n}\right\}_{n \in N}$ both have 0 as the $s$-accumulation (resp. SR-accumulation) point. But 0 is not a $T_{\mathrm{RC}}$-accumulation (resp. $T_{\mathrm{SR}}$-accumulation) point of $\left\{x_{n}\right\}_{n \in N}$ or $\Im$.

Theorem 2.9. A topological space $(X, T)$ is $S$-closed iff $\left(X, T_{R C}\right)$ is compact.

Proof. Let $(X, T)$ be $S$-closed. Then every regular closed cover of $X$ has a finite subcover. But the collection of all regular closed sets of $(X, T)$ is a subbase for $T_{\mathrm{RC}}$. Therefore every subbasic open cover of $\left(X, T_{\mathrm{RC}}\right)$ has a finite subcover. By Alexander subbase theorem, $\left(X, T_{\mathrm{RC}}\right)$ is compact.

Conversely, let $\left(X, T_{\mathrm{RC}}\right)$ be compact. Since $\mathrm{RC}(X) \subset T_{\mathrm{RC}}$, every regular closed cover of $(X, T)$ has a finite subcover. So $(X, T)$ is $S$-closed by [Theorem 1 of Asha Mathur [8]].

Theorem 2.10. A topological space $(X, T)$ is s-closed iff $\left(X, T_{\mathrm{SR}}\right)$ is compact.

Proof. It is similar to Theorem 2.9 and is thus omitted.

The following theorem for $S$-closed spaces improves Theroem 1 of Asha Mathur [8], Theorem 1.3 of T. Noiri [9] and Theorem 2 of Thompson [11]; and the theorem for $s$-closed spaces improves proposition 3.1 of Maio and Noiri [7].

Theorem 2.11. Let $(X, T)$ be a topological space. Then the following are equivalent. 
i) $(X, T)$ is $S$-closed (resp. s-closed)

ii) every proper regular open (resp. Semi-regular) set is an $S$-set (resp. s-set) in $(X, T)$.

iii) every closed set of $\left(X, T_{R C}\right)\left[\right.$ resp. $\left.\left(X, T_{S R}\right)\right]$ is an $S$-set (resp. s-set) in $(X, T)$.

iv) every family of regular open (resp. Semi-regular) subsets of $(X, T)$ with the finite intersection property (f.i.p. for short) has non-void intersection.

v) every family of closed subsets of $\left(X, T_{R C}\right)\left[\right.$ resp. $\left.\left(X, T_{S R}\right)\right]$ with the f.i.p. has nonvoid intersection.

vi) every filter base in $(X, T)$ has an s-accumulation (resp. SR-accumulation) point.

vii) every net in $(X, T)$ has an s-accumulation (resp. SR-accumulation) point.

viii) every filter base in $(X, T)$ has a $T_{R C}$-accumulation (resp. $T_{S R}$-accumulation) point.

ix) every net in $(X, T)$ has a $T_{R C}$-accumulation (resp. $T_{S R}$-accumulation) point.

$\mathrm{x})$ every net in $(X, T)$ has a $T_{R C}$-convergent (resp. $T_{R S}$ convergent) subnet.

xi) every filter $\Im$ in $(X, T)$ has a sub-ordinate filter $\Im_{0}$ of $\Im$ which is $T_{R C}$-convergent (resp. $T_{S R}$-convergent).

xii) every universal net in $(X, T)$ is $T_{R C}$-convergent (resp. $T_{S R}$-convergent).

xiii) every ultrafilter in $(X, T)$ is $T_{R C}$-convergent (resp. $T_{S R}$-convergent).

Proof. The facts discussed above prove the theorem immediately.

Definition 2.12. A function $f:(X, T) \rightarrow\left(Y, T^{\prime}\right)$ is said to be $(\theta, s)$-continuous [5] (resp. $\gamma$-continuous [3]) if for each $x \in X$ and each $W \in \mathrm{SO}(f(x))$, there is an open set $V$ containing $x$ such that $f(V) \subset \operatorname{cl} W($ resp. $f(V) \subset \operatorname{scl} W)$.

Since $\operatorname{RC}(x)=\{\operatorname{cl} W: W \in \mathrm{SO}(x)\}[5]($ resp. $\mathrm{SR}(x)=\{\operatorname{scl} W: W \in \mathrm{SO}(x)\}[7])$, the above definition can equivalently be stated as: a function $f:(X, T) \rightarrow\left(Y, T^{\prime}\right)$ is $(\theta, s)$-continuous (resp. $\gamma$-continuous) iff $f^{-1}(W)$ is open in $X$, for every $W \in \operatorname{RC}(Y)$ (resp. $W \in \operatorname{SR}(Y)$ ).

Theorem 2.13. A topological space $(X, T)$ is $S$-closed iff it is a $(\theta, s)$-continuous image of a compact space.

Proof. Let $(X, T)$ be $S$-closed. Then by Theorem $2.9,\left(X, T_{\mathrm{RC}}\right)$ is compact. Let $i:\left(X, T_{\mathrm{RC}}\right) \rightarrow(X, T)$ be the identity function, which is obviously $(\theta, s)$-continuous. Therefore there exist a compact space and a $(\theta, s)$-continuous function such that the $S$-closed space $(X, T)$ is the $(\theta, s)$-continuous image of a compact space.

Conversely, let $f:\left(Y, T^{*}\right) \rightarrow(X, T)$ be $(\theta, s)$-continuous surjection and $\left(Y, T^{*}\right)$ be compact. Let $\left\{V_{\alpha}: \alpha \in I\right\}$ be a cover of $(X, T)$ be regular closed sets of $(X, T)$. Then $\left\{f^{-1}\left(V_{\alpha}\right): \alpha \in I\right\}$ is a cover of the compact space $\left(Y, T^{*}\right)$ by open sets of $\left(Y, T^{*}\right)$. Therefore there exists a finite subset $I_{0}$ of $I$ such that $\left\{f^{-1}\left(V_{\alpha}\right): \alpha \in I_{0}\right\}$ covers $Y$ and hence $\left\{V_{\alpha}: \alpha \in I_{0}\right\}$ covers $X$. Therefore $(X, T)$ is $S$-closed.

Theorem 2.14. A topological space $(X, T)$ is s-closed iff it is a $\gamma$-continuous image of a compact space.

Proof. The proof is similarl to Theorem 2.13 and thus omitted. 
Maio and Norir [7] initiated quasi-irresolute function and established that such functions preserve $s$-sets. Here we introduce a weaker form of quasi-irresolute function which also has the same property.

Definition 2.15. ([7]) A function $f: X \rightarrow Y$ is said to be quasi-irresolute if for each $x \in X$ and each $V \in \mathrm{SO}(f(x))$ there exists a $U \in \mathrm{SO}(x)$ such that $f(U) \subset \operatorname{scl} V$.

Definition 2.16. A function $f:(X, T) \rightarrow\left(Y, T^{\prime}\right)$ is said to be weakly quasi-irresolute if $f:\left(X, T_{\mathrm{SR}}\right) \rightarrow\left(Y, T_{\mathrm{SR}}^{\prime}\right)$ is continuous.

Theorem 2.17. Every quasi-irresolute function is weakly quasi-irresolute.

Proof. Let $f:(X, T) \rightarrow\left(Y, T^{\prime}\right)$ be quasi-irresolute. Then $f^{-1}(V)$ is semi-regular set in $(X, T)$ for every $V \in \mathrm{SR}(Y)$. But the collection of all semi-regular sets of $\left(Y, T^{\prime}\right)$ is a subbase for $\left(Y, T_{\mathrm{SR}}^{\prime}\right)$. Hence $f:\left(X, T_{\mathrm{SR}}\right) \rightarrow\left(Y, T_{\mathrm{SR}}^{\prime}\right)$ is continuous. Therefore $f:(X, T) \rightarrow\left(Y, T^{\prime}\right)$ is weakly quasi-irresolute.

Remark 2.18. That the coverse of the above theorem is not necessarily true follows from the following example.

Example 2.19. Let $f: R \rightarrow R$ where $R$ is the set of reals with the usual topology $T$ be a function defined by

$$
f(x)= \begin{cases}r_{1} & \text { if } x \text { is rational, } \\ r_{2} & \text { if } x \text { is irrational, with } r_{2}>r_{1}>0 .\end{cases}
$$

Clearly $T_{\mathrm{SR}}$ is the discrete topology; therefore $f:\left(R, T_{\mathrm{SR}}\right) \rightarrow\left(R, T_{\mathrm{SR}}\right)$ is continuous and hence $f$ is weakly quasi-irresolute function. But $f$ is not a quasi-irresolute function. In fact if $\delta$ be such that $0<\delta<\left|\left(r_{2}-r_{1}\right) / 2\right|$, then the open interval $J=\left(r_{2}-\delta, r_{2}+\delta\right)$ is a semi-regular set in $(R, T)$; but $f^{-1}(J)$ is the set of all irrationals of $R$. Hence $f^{-1}(J)$ is not even a semi-open set in $R$.

Hence we get an improved result of Corollary 5.1 of Maio and Noiri [7].

Theorem 2.20. If $f:(X, T) \rightarrow\left(Y, T^{\prime}\right)$ is weakly quasi-irresolute and $K$ is an s-set of $(X, T)$, then $f(K)$ is an s-set in $Y$.

Proof. Let $\left\{U_{\alpha}: \alpha \in I\right\}$ be a cover of $f(K)$ by semi-regular sets of $\left(Y, T^{\prime}\right)$. Since $f:(X, T) \rightarrow\left(Y, T^{\prime}\right)$ is weakly quasi-irresolute, $\left\{f^{-1}\left(U_{\alpha}\right): \alpha \in I\right\}$ is a cover of $K$ by open sets of $\left(X, T_{\mathrm{SR}}\right)$. By Theorem $2.10, K$ is compact in $\left(X, T_{\mathrm{SR}}\right)$. Therefore, there exists a finite subset $I_{0}$ of $I$ such that $K \subset \cup\left\{f^{-1}\left(U_{\alpha}\right): \alpha \in I_{0}\right\}$. Which implies $f(K) \subset \cup_{\alpha \in I_{0}} U_{\alpha}$. Therefore $f(K)$ is an $s$-set in $Y$.

Corollary 2.21. If $f:(X, T) \rightarrow\left(Y, T^{\prime}\right)$ is weakly quasi-irresolute surjection and $(X, T)$ is s-closed then $\left(Y, T^{\prime}\right)$ is also s-closed.

Definition 2.22. ([7]) A space $(X, T)$ is said to be weakly Hausdorff if every point of $X$ is the intersection of regular closed sets of $X$. 
The following theorem improves Corollary 5.2 of Maio and Noiri [7].

Theorem 2.23. Let $f:(X, T) \rightarrow\left(Y, T^{\prime}\right)$ be weakly quasi-irresolute, $(X, T)$ is $s$ closed and $\left(Y, T^{\prime}\right)$ be weakly Hausdorff. Then the image of each semi- $\theta$-closed set [7] in $X$ is semi- $\theta$-closed in $Y$.

Proof. Let $K$ be a semi- $\theta$-closed set in $(X, T)$. Then by Proposition 4.2 of Maio and Noiri [7], $K$ is an $s$-set in $X$. By Theorem 2.20, $f(K)$ is an $s$-set in $Y$. Therefore by Proposition 4.3 of Maio and Noiri [7], $f(K)$ is semi- $\theta$-closed set in $(Y, T)$.

\section{Anti-S-Closed and Anti-s-Closed Spaces}

P. Bankston [1] studied topological anti-properties. Reilly \& Vamanamurthy [10] extended these concepts to semi-compact spaces. In a similar fashion [10], here we introduce and characterize two new topological anti-properties under the terminology 'anti- $S$-closedness' and 'anti-s-closedness' along with their mutual relationships.

Definition 3.1. A topological space $(X, T)$ is said to be anti-S-closed (resp. anti-s -closed) if only the finite subsets of $(X, T)$ are $S$-sets (resp. $s$-sets) of $(X, T)$.

An infinite subset $A$ of $(X, T)$ is said to be anti- $S$-closed (resp. anti-s-closed) relative to $X$ if only the finite subsets of $A$ are $S$-sets (resp. $s$-sets) in $(X, T)$.

Theorem 3.2. A topological space $(X, T)$ is anti-S-closed iff for every infinite set $N$ of $X$ and each point $x$ of $X$, there exists a regular closed set $R$ containing $x$ such that $N \backslash R$ is not an $S$-set in $(X, T)$.

Proof. Let the given condition hold. We have to show that $(X, T)$ is anti- $S$-closed. Let $N$ be any infinite set and let $x \in X$. Then by hypothesis, there exists a $R \in R C(x)$ such that $N \backslash R$ is not an $S$-set. Therefore there exists a cover $\mathcal{A}$ of $N \backslash R$ by regular closed sets of $X$ which has no finite subcover. So $N$ is not an $S$-set in $(X, T)$. Therefore $(X, T)$ is anti- $S$-closed.

Conversely, let $(X, T)$ be anti- $S$-closed space. Let $N$ be any infinite subset of $X$ and let $x$ be any point of $X$. Then by definition of anti-S-closed space, $N$ and hence $N \cup\{x\}$ is not an $S$-set. Therefore there exists a cover $\mathcal{A}$ of $N \cup\{x\}$ by regular closed sets which has no finite subcover. Hence there exists a member $R \in \mathcal{A}$ such that $x \in R$. So $N \backslash R$ is not an $S$-set in $(X, T)$.

Theorem 3.3. A topological space $(X, T)$ is anti-s-closed iff for every infinite set $N$ of $X$ and each point $x$ of $X$, there exists a $V \in S R(x)$ such that $N \backslash V$ is not an s-set in $(X, T)$.

Proof. The proof is similar to that of the above theorem.

Theorem 3.4. If $(X, T)$ is anti-S-closed then it is anti-s-closed. 
Proof. The proof immediately follows because of the fact that every $s$-set is an $S$-set.

Remark 3.5. That the converse of the above theorem is not necessarily true follows from the following example.

Example 3.6. Let $X$ be set of all integers with the topology $T$ having the base $\{X,\{0\},\{-1\},\{-2\}, \ldots\}$. Then no infinite set of $X$ is an $s$-set; if we have $Z^{+}$, the set of positive integers, then $\{\{0,1\},\{0,2\},\{0,3\}, \ldots\}$ is a semi-open cover of $Z^{+}$and scl $\{0, n\}=\{0, n\}$. Then $Z^{+}$is not an $s$-set. But $Z^{+}$is an $S$-set; if we consider $Z-Z^{+}$then $\{\{0\},\{-1\},\{-2\}, \ldots\}$ is a semi-open cover of $Z-Z^{+}$and $\operatorname{scl}\{-n\}=\{-n\}$. Therefore it has no finite subcover. So $Z-Z^{+}$is not an $s$-set. If $L \subset X$ be such that it is infinite and contains finitely many points from $Z^{+}$then again this can be shown to be a non $s$-set; if it contains infinitely many elements from $Z^{+}$, the same thing happens. Thus $X$ is anti-s-closed but not anti-S-closed.

Theorem 3.7. Any topological space $(X, T)$ which is not $S$-closed (resp. not sclosed) has a proper infinite subset which is anti-S-closed (resp. anti-s-closed) relative to $X$.

Proof. Since $(X, T)$ is not $S$-closed (resp. not $s$-closed), there exists, in particular, a countable cover $\mathcal{A}$ of $X$ by regular closed (resp. semi-regular) sets which has no finite subcover. We pick up the points $x_{m+1} \in X-\cup_{i=1}^{n} V_{i}$ (where $V_{i} \in \mathcal{A}$ ). Then the set $\left\{x_{m}, m \in N\right\}$, where $N$ is the set of naturals, is a proper infinite subset of $X$ which is not $S$-set (resp. s-set). Therefore every infinite subset of $\left\{x_{m}: m \in N\right\}$ is not an $S$-set (resp. $s$-set) in $(X, T)$. Hence the infinite subset $\left\{x_{m}: m \in N\right\}$ is anti-S-closed (resp. anti-s-closed) relative to $X$.

Definition 3.8. A topological space $(X, T)$ is said to be hereditarily $S$-closed (resp. hereditarily $s$-closed) if each of its subsets is $S$-set (resp. $s$-set) in $(X, T)$.

Theorem 3.9. A topological space $(X, T)$ is hereditarily $S$-closed (resp. hereditarily s-closed) iff $(X, T)$ is anti-(anti-S-closed) [resp. anti-(anti-s-closed)].

Proof. Let $(X, T)$ be anti-(anti- $S$-closed) [resp. anti-(anti-s-closed)]. If possible let $(X, T)$ be not hereditarily $S$-closed (resp. hereditarily $s$-closed). Then there exists a subset $B$ of $X$ such that $B$ is not an $S$-set (resp. $s$-set) and hence $B$ must be infinite. Therefore by Theorem 3.7, $B$ has an infinite subset $M$ which is anti- $S$-closed (resp. anti$s$-closed) relative to $X$-a contradiction to the definition of anti-(anti- $S$ - closed) [resp. anti(anti-s-closed)].

Coversely, let $(X, T)$ be hereditarily $S$-closed (resp. hereditarily $s$-closed). If possible, let $(X, T)$ be not anti-(anti- $S$-closed) [resp. anti-(anti-s-closed)]. Then by definition there exists an infinite subset $V$ of $X$ which is anti- $S$-closed (resp. anti-s-closed) relative to $X$. Therefore $V$ is not an $S$-set (resp. $s$-set) in $(X, T)$-a contradiction. 


\section{Acknowledgements}

The author gratefully acknowledges the learned referee for his constructive suggestions which improved the paper to a great extent.

\section{References}

[1] P. Bankston, The total negation of a topological property, Illinois J. Math. 23(1979), 241252.

[2] N. Biswas, On characterizations of semi-continuous functions, Atti Accad. Nat. Lincei Rend. Cl. Sci. Fis. Mat. Natur 48(1970), 399-402.

[3] S. Ganguly and C. K. Basu, More on s-closed spaces, Soochow J. Math. 18(1992), 409-418.

[4] M. Ganster and I. L. Reilly, A note on s-closed spaces, Indian J. Pure Appl. Math. 19(1988), 1031-1033.

[5] J. E. Joseph and M. Kwack, On S-closed spaces, Proc. Amer. Math. Soc. 80(1980), 341348.

[6] N. Levine, Semi-open sets and semi-continuity in topological spaces, Amer. Math. Monthly 70(1963), 36-41.

[7] G. D. Maio and T. Noiri, On s-closed spaces, Indian J. Pure Appl. Math. 18(1987), 226-233.

[8] A. Mathur, A note on S-closed spaces, Proc. Amer. Math. Soc. 74(1979), 350-352.

[9] T. Noiri, On S-closed spaces, Ann. Dela Soc. Sci. Sci. de Bruxelles T. 91 IV(1977), 189-194.

[10] I. L. Reilly and M. K. Vamanamurthy, On semi-compact spaces, Bull. Malaysian Math. Soc. 2(1984), 61-67.

[11] T. Thompson, S-closed spaces, Proc. Amer. Math. Soc. 60(1976), 335-338.

Department of Mathematics, University of Kalyani, Kalyani, Dist.-Nadia, West Bengal, Pin741235 , India.

E-mail: cubasu@klyunivernet.in 